\title{
Mise en oeuvre d'un modèle réduit physique du port de TOGA (Corse). Comparaison des mesures de l'agitation du plan d'eau avec les résultats de simulations numériques en houles régulière et irrégulière.
}

\author{
David Lajoie, \\ Ingénieur société ACRI \\ René Bonnefille \\ Docteur ès Sciences
}

\section{Résumé}

Suite aux dégâts subis par le port de Toga (Corse) en 1990, il a été décidé d'explorer les diverses interventions qui seraient susceptibles d'améliorer le confort du plan d'eau. Les études ont été réalisées suivant une première phase numérique destinée à dégager une solution viable du point de vue de l'agitation résiduelle, puis une deuxième phase de validation de cette solution par des tests en cuve à houle permettant aussi de parachever la constitution des ouvrages du point de vue de la stabilité. Dans ce cas précis, où le port renferme de nombreux quais verticaux favorisant la résonance des oscillations du plan d'eau, on montre l'intérêt de simuler l'agitation en considérant des houles incidentes irrégulières en fréquence plutôt que monochromatiques. Les résultats de calcul sont ainsi en bon accord avec les mesures statistiques réalisées en cuve à houle.

\section{Introduction}

Le port de Toga (Haute Corse) est actuellement le siège de fortes agitations générées par les houles de tempête. En décembre 1990, pour des conditions de tempête jugées relativement peu exceptionnelles, plusieurs déficiences telles que des franchissements importants et une agitation résiduelle au-delà des limites habituellement admises sont apparues, mettant en doute la fiabilité du port.

Des études ont été lancées à l'initiative de la Société du Port de Toga Plaisance afin de définir les modifications nécessaires à apporter sur les ouvrages existants pour assurer complètement le confort et la sécurité du port. L'étude hydraulique a comporté deux phases :

- une première phase de préqualification, fondée sur des simulations numériques du niveau d'agitation portuaire, visant à comparer l'efficacité de piusieurs configurations modifiées par rapport à la situation actuelle du port. Cette phase a permis de conclure sur l'intérêt de prolonger de $60 \mathrm{~m}$ le brise-lames existant.

- une seconde phase d'essais en modèle réduit physique (réalisée au laboratoire HR Wallingford, Angleterre) ayant pour objectif de valider la solution choisie des points de vue de l'agitation résiduelle, des franchissements et de la stabilité des ouvrages.

On présente dans cette communication l'approche numérique du calcul de l'agitation en comparant les résultats issus de simulations classiques en houle régulière avec ceux plus réalistes tenant compte de la répartition de l'énergie de la houle incidente sur une bande de fréquences. Les résultats de simulation en houle irrégulière sont ensuite confrontés aux mesures de hauteurs de houles significatives en plusieurs points du modèle réduit physique. 


\section{L'agitation dans le port de TOGA}

Le port de Toga est situé dans le prolongement de la partie Nord du port de Commerce de Bastia. Il est ouvert vers le Nord, ce qui d'un point de vue agitation le rend particulièrement vulnérable aux houles provenant du secteur Nord-Est. Ces houles sont d'autant plus dangereuses qu'un haut-fond situé devant le port favorise leur convergence vers la passe du port (Cf. figure 1 ci-après).

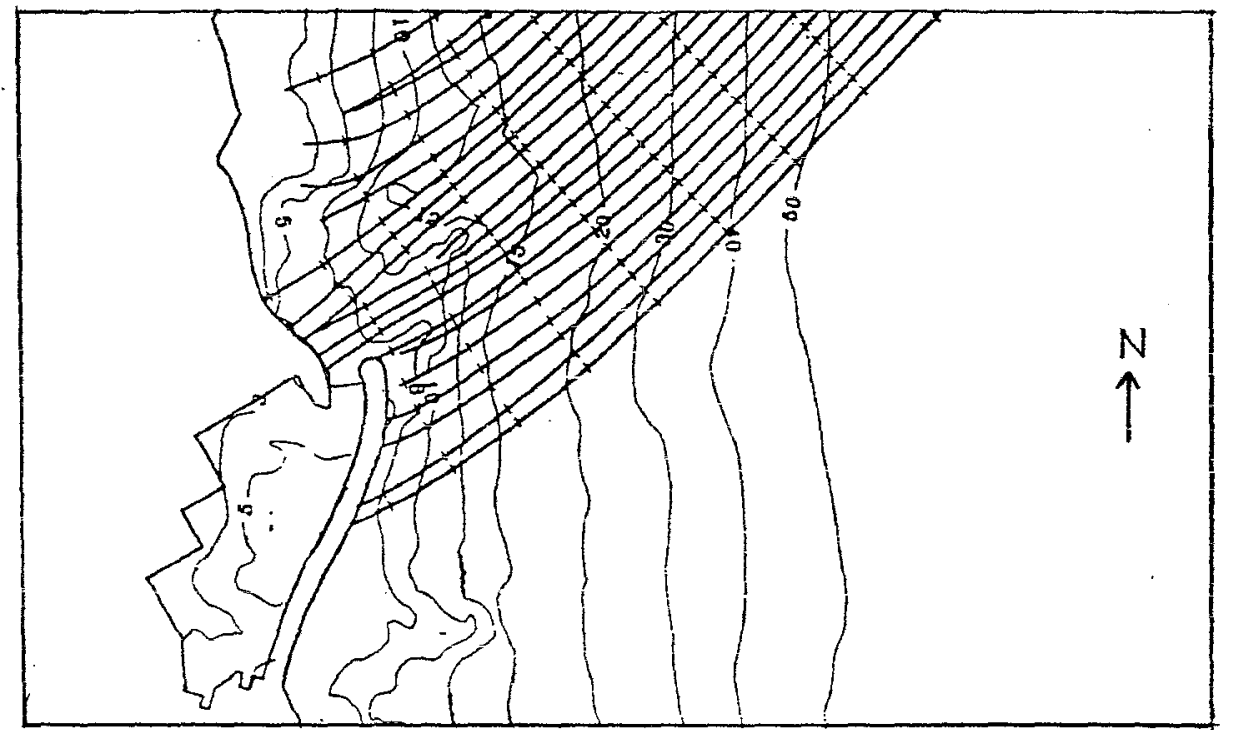

Figure I: Vue d'ensemble du port. Plan de vague pour une houle de Nord-Est. $T=7,5 \mathrm{~s}$

\subsection{Le modèle numérique du port}

L'agitation à l'intérieur du port a été simulée en exploitant le logiciel SMMAC, développé par ACRI. Le domaine d'étude, très vaste, a été divisé en deux régions pour lesquelles on a adopté des hypothèses simplificatrices différentes, sans pour autant violer fondamentalement la réalité physique :

- la région s'étendant du large vers le port, sur laquelle on simule la propagation de la houle incidente influencée uniquement par de faibles variations du fond ;

- le port et ses abords, où l'on tient compte des faibles variations du fond et des ouvrages et présence.

La propagation de la houle du large vers la côte a été simulée en employant une méthode classique de raies, fondée sur la théorie de la réfraction pure de la houle. La bathymétrie au large étant assez régulière pour ne pas provoquer de convergence faisant diffracter la houle, cette approche s'avère suffisante.

Le champ de vagues dans la zone portuaire a été calculé en résolvant l'équation de diffractionréfraction combinée de Berkhoff [1], laquelle s'applique dans le cadre de la théorie linéaire des ondes monochromatiques se propageant sur des fonds faiblement variables. Cette dernière hypothèse permet d'exprimer le potentiel des vitesses $\Phi$ en dissociant les variations verticales et horizontales. Soit "j" la pente moyenne du fond, le potentiel au premier ordre suivant $O(j z)$ s'écrit ainsi de la façon suivante :

$$
\Phi(\mathrm{x}, \mathrm{y}, \mathrm{z}, \mathrm{t})=\Re \mathrm{e}\left[\varphi(\mathrm{x}, \mathrm{y}) \frac{\mathrm{ch}[\mathrm{k}(\mathrm{z}+\mathrm{h})]}{\operatorname{ch}(\mathrm{kh})} \mathrm{e}^{-\mathrm{i} \omega \mathrm{t}}\right]
$$

avec $h(x, y)$ : la profondeur ; $k(x, y)$ : le nombre d'onde ; $\omega$ : la pulsation. 
L'équation d'onde de Berkhoff, établie en introduisant les variations au deuxième ordre du potentiel $\Phi$ par rapport à $O(j z)$ s'exprime comme suit [1] :

$$
\operatorname{div}\left(\mathrm{cc}_{\mathrm{g}} \overrightarrow{\operatorname{grad} \varphi}\right)+\mathrm{cc}_{\mathrm{g}} \mathrm{k}^{2} \varphi=0
$$

avec : $c$ : la vitesse de phase $; c_{g}$ : la vitesse de groupe.

Cette équation, de type elliptique, a été résolue par une méthode d'éléments finis sur toute la zone portuaire et une partie de ses abords. On a pris soin d'englober la bosse située devant la passe dans la zone d'étude, de façon à prendre en compte le phénomène de diffraction sur toute cette zone de convergence de la houle.

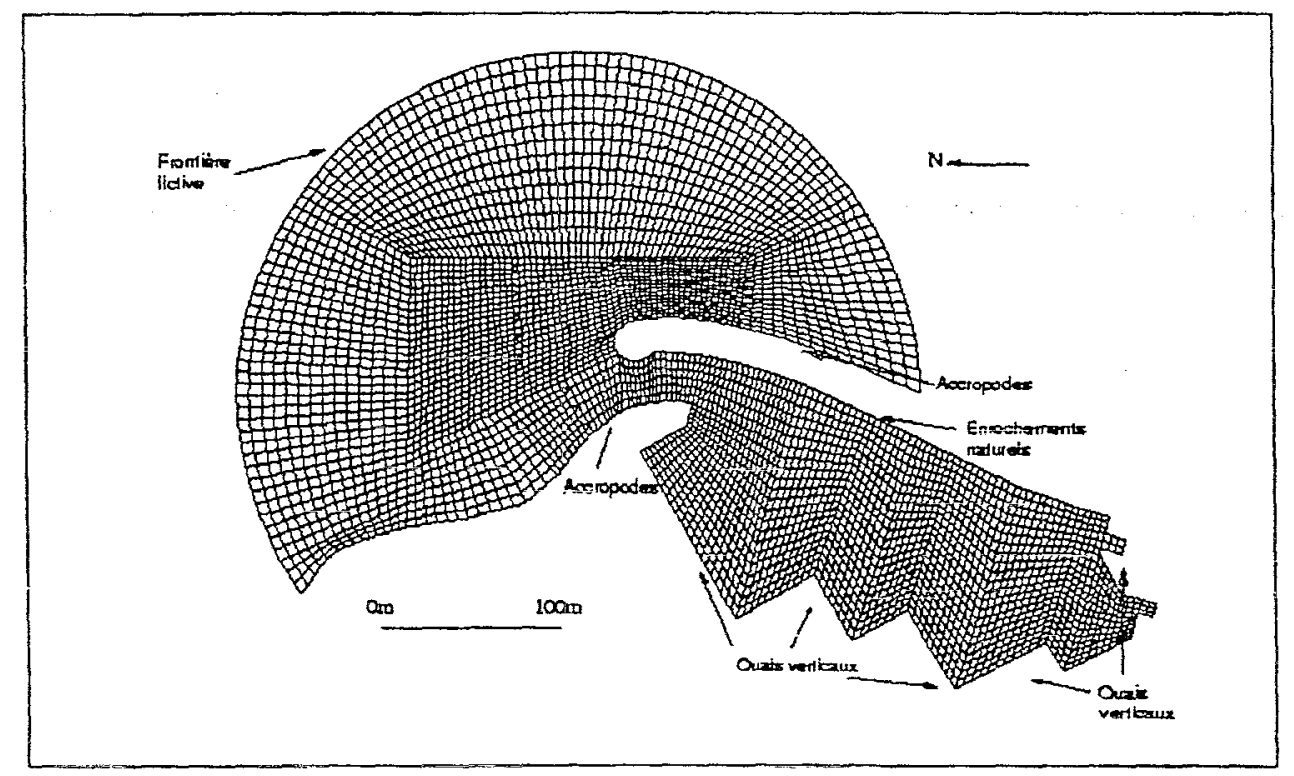

Figure 2 : Maillage du port de Toga (configuration actuelle)

Les frontières du modèle sur lesquelles sont appliquées les conditions aux limites du problème aux éléments finis sont de deux types :

- les frontières réfléchissantes représentant les digues ou les quais, sur lesquelles on applique un coefficient de réflexion $r$, en accord avec la dissipation subie par la vague d'incidence $\theta$ sur l'ouvrage. Il vient ainsi $\frac{\partial \varphi}{\partial n}=i k \cos \theta \frac{1-r}{1+r} \varphi$. L'ordre de grandeur du coefficient $r$ est de 0,4 à 0,6 pour une digue à talus et de 0,95 à 1 pour un quai vertical.

- la frontière radiative, qui ne correspond pas à une frontière physique, sur laquelle on affiche les caractéristiques de la houle incidente interpolées à partir des résultats du modèle par raies, ainsi qu'une condition de transmission des ondes de retour (celles réfléchies sur les côtés extérieurs des digues ou celles diffractant vers le large à travers la passe du port) [3].

Les effets de franchissement des digues, très délicats à modéliser, ne sont pas pris en compte. 


\subsection{Résultats dans la configuration actuelle du port}

\subsubsection{Simulations en houle régulière}

Un premier diagnostic sur le comportement du port actuel a été fait en multipliant les simulations en houles monochromatiques pour la provenance de houle au large jugée néfaste : Nord-Est. La théorie employée étant linéaire, la succession de simulations espacées de $0,1 \mathrm{~s}$ en période a permis de dresser des fonctions de transfert $A(x, y, \omega)$ reliant la hauteur de houle au large $\mathrm{H}_{0}(\omega)$ à la hauteur $\mathrm{H}(\mathrm{x}, \mathrm{y})$ en tout point du port de telle sorte que :

$$
A(x, y, \omega)=\frac{H(x, y, \omega)}{H_{0}(\omega)}
$$

Exceptée la pârtie intérieure de la digue principale, constituée d'enrochements naturels, le port de Toga est uniquement délimité par des quais verticaux, ce qui favorise fortement l'apparition de résonances dans les bassins. Il en résulte de fortes irrégularités dans les réponses spectrales $A(x, y, w)$ de chaque zone du port. Sur la figure 3 , on présente la fonction de transfert moyenne sur une zone jugée critique, couvrant le fond du premier bassin du port (zone entourant le point de mesure $10, \mathrm{Cf}$. figure 8).

Le balayage en périodes permet d'identifier les fréquences propres de plusieurs zones du port. Ici, le fond du premier bassin est le siège d'une forte résonance aux alentours de $T=11 \mathrm{~s}$ avec un pic à $T=11,2 \mathrm{~s}$. Pour cette période très néfaste, estimée centenale, on montre sur la figure 4 le champ de facteur d'amplification par rapport à la hauteur de houle au large.

La résonance vers $T=11,2 \mathrm{~s}$ est très marquée dans tout le premier bassin, dont la taille correspond précisément 2 longueurs d'onde. La houle peut y atteindre localement 1 à 1,5 fois la houle incidente, ce qui est bien sûr considérable. Cette approche en houle régulière est toutefois pessimiste. On montre en effet que les plages de fréquences de résonance à l'intérieur du port sont très étroites, si bien qu'une houle monochromatique de fréquence égale à l'une des périodes propres du port génère de fortes agitations en certaines zones, alors que ces mêmes zones sont très peu agitées pour une fréquence voisine. Or, la houle réelle étant irrégulière en fréquences, le phénomène de résonance est en réalité largement plus atténué.

\subsubsection{Simulations en houle irrégulière}

Afin de quantifier de façon plus réaliste le niveau d'agitation dans un port sujet à des résonances pointues, nous avons simulé les effets d'une houle dont l'énergie est répartie sur plusieurs fréquences. Le spectre fréquentiel choisi pour la houle incidente est également celui qui a été adopté lors des essais en cuve à houle, à savoir le spectre de JONSWAP avec un coefficient d'élancement $\gamma=3,3$. La densité spectrale d'énergie de la houle incidente est définie comme suit :

$$
\mathrm{S}_{0}(\omega)=\alpha \mathrm{H}_{\mathrm{S}}^{2} \omega_{\mathrm{p}}^{4} \omega^{-5} \mathrm{e}^{-1,25\left(\frac{\omega}{\omega_{\mathrm{p}}}\right)^{-4}} \gamma^{\mathrm{a}} ; \mathrm{a}=\mathrm{e}^{-\frac{\left(\omega-\omega_{\mathrm{p}}\right)^{2}}{2 \sigma^{2} \omega_{\mathrm{p}}^{2}}}
$$

avec $\omega_{\mathrm{p}}$ : pulsation pic $; \sigma=0,07$ pour $\omega<\omega_{\mathrm{p}}$ et $\sigma=0,09$ pour $\omega>\omega_{\mathrm{p}}$;

$\alpha$, étant une constante telle que la hauteur significative $H_{s}$ puisse vérifier la loi classique inhérente aux processus supposés gaussiens (et dont la densité de probabilité des maxima suit elle-même une loi de Rayleigh [2]) :

$$
H_{S}=4 \sqrt{b_{0}^{\infty} S_{0}(\omega) d \omega}
$$


Les simulations en houle irrégulière font intervenir les fonctions de transfert $A(x, y, \omega)$ en tout point du port. Sous les hypothèses de la théorie linéaire, on peut définir une densité spectrale d'énergie $S(x, y, \omega)$ en tant que réponse du port par rapport à la densité d'énergie au large $S_{0}(\omega)$ de la façon suivante :

$$
S(\omega, x, y)=A^{2}(\omega, x, y) \cdot S_{0}(\omega)
$$

Sur la figure 5 , on a fait apparaître le champ de facteur d'amplification a(x,y) en terme de hauteurs de houle statistiques tel que :

$$
a(x, y)=\frac{\sqrt{\int_{0}^{\infty} S(\omega, x, y) d \omega}}{\sqrt{\int_{0}^{\infty} S_{0}(\omega) d \omega}}
$$

$\mathrm{S}_{0}(\omega)$ étant la densité spectrale au large suivant les critères exprimés ci-avant pour une période pic de $11,2 \mathrm{~s}$.

Par rapport à la simulation en houle monochromatique de période $11,2 \mathrm{~s}$, les résultats de la figure 5 montrent un champ de vagues plus lissé, beaucoup plus proche de ce que l'on remarque en réalité. Le coefficient d'amplification est de l'ordre de $25 \%$ à $35 \%$ au lieu de $100 \%$ à $150 \%$. Dans ces conditions de houles extrêmes (environ centenale), où la houle significative au large dépasse les $6,5 \mathrm{~m}$, cela représente une agitation résiduelle supérieure à $2 \mathrm{~m}$, encore considérable. Cette hauteur a été mesurée lors des tests effectués au. HR Wallingford.

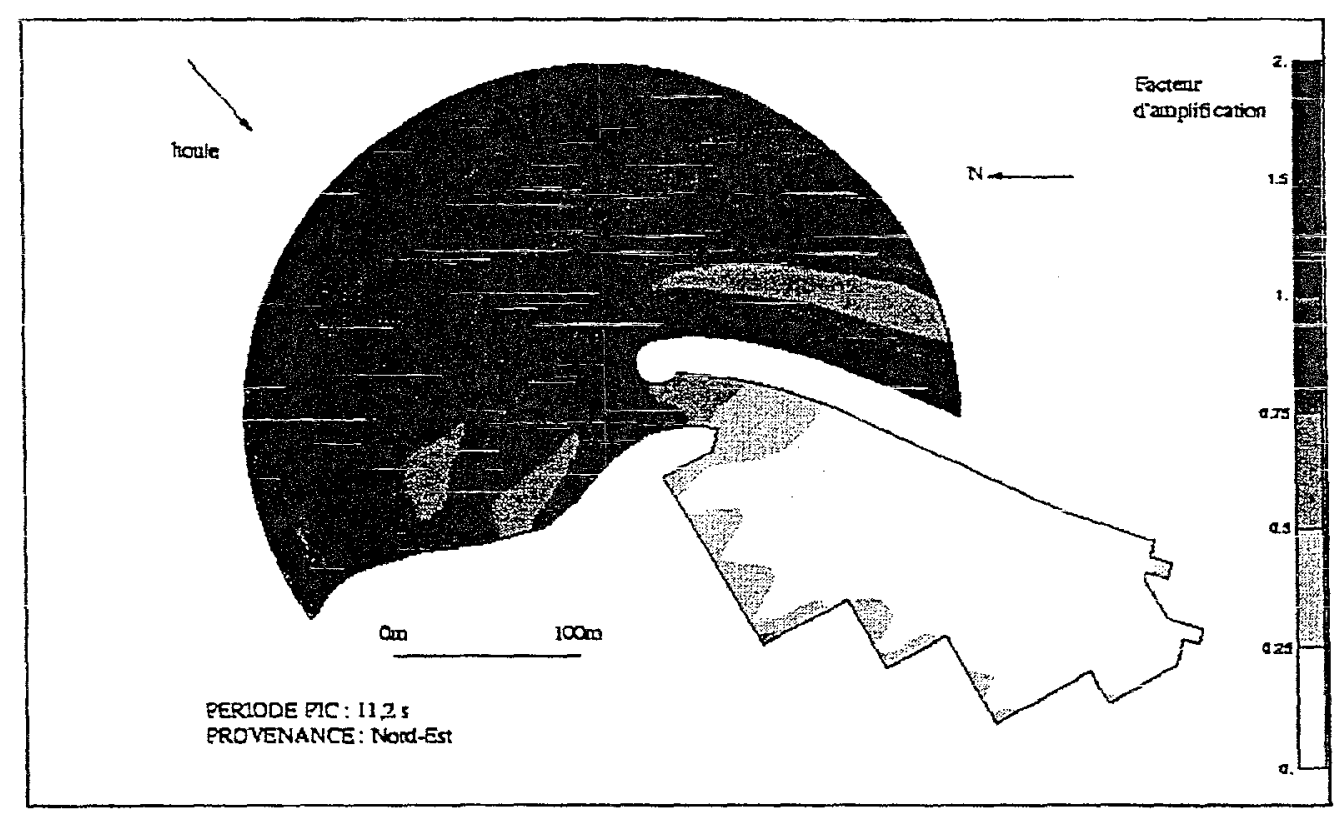

Figure 5 : Facteur d'amplification par rapport à la hauteur au large. Configuration actuelle. Houle irrégulière. Tp=11,2s. Provenance : NE 


\subsection{Configuration future}

Les nouvelles dispositions envisagées étaient les suivantes :

- la construction d'une contre-jetée au Nord ;

- la mise en place d'un obstacle émergeant au niveau de la zone convergence ;

- l'allongement de la digue principale.

Les différentes simulations et balayages en périodes nous ont amenés à opter pour un allongement de $60 \mathrm{~m}$ de la digue principale, qui permet de réduire d'un facteur 2 à 3 l'agitation résiduelle actuelle (Cf. figure 3 ).

Les simulations en houles monochromatiques ont laissé apparaitre un niveau général d'agitation relative dans le port modifié de l'ordre de $3 \%$ à $10 \%$ (soit $15 \mathrm{~cm}$ à $60 \mathrm{~cm}$ en situations extrêmes), avec toutefois queiques résonances locales beaucoup plus élevées généralement près des quais. Les essais en cuve et les simulations en houle irrégulière montrent néanmoins que ces fortes agitations locales sont largement amoindries par la dispersion en fréquences.

Sur les figures 6 et 7 , on présente les facteurs d'amplification pour une houle estimée vingtenale de période pic $7,5 \mathrm{~s}$ et de $\mathrm{H}_{\mathrm{s}}=5,7 \mathrm{~m}$, laquelle occasionne peu de franchissement dans la noüvelle configuration du port. L'agitation en houle irrégulière, de l'ordre de $10 \%$ à $15 \%$ de la hauteur au large (et jusqu'à $20 \%$ dans les coins) dans la configuration actuelle, n'atteint plus que $3 \%$ à $7 \%$ (et moins de $10 \%$ dans les coins) dans la configuration future.

Pour cette même houle, sur la figure 9 , on compare les hauteurs significatives mesurées en différents points du port modifié (points 7 à 15 sur la figure 8) et les fourchettes haute et basse des valeurs relevées sur les mêmes zones d'après les simulations. Les hauteurs numériques ont été calculées en admettant en tout point du port la relation $H_{S}(x, y)=4 \sqrt{\int_{6}^{\infty} S(\omega, x, y) d \omega}$, liée aux processus gaussiens. En adoptant des coefficients de réflexion égaux à 0,99 sur les quais verticaux et 0,4 sur la digue à talus, les résultats numériques sont en très bon accord avec les résultats expérimentaux.

Pour une houle vingtenale, l'agitation ne dépasse pas $60 \mathrm{~cm}$, restant généralement inférieur à $30 \mathrm{~cm}$, ce qui est encore sécurisant. Les essais ont montré que pour les houles plus extrêmes (centenales) pouvant franchir les ouvrages et générant les résonances dans le premier bassin, l'agitation pouvait atteindre $1 \mathrm{~m}$ au point 10 , occasionnant dans ce cas quelques dommages.

\section{La stabilité des ouvrages}

Les essais en cuve ont permis de parachever la constitution des ouvrages. Afin de limiter les franchissements et d'assurer la stabilité des ouvrages (ou du moins leur non mise en péril sous les tempêtes les plus extrêmes), les ouvrages existants ont dû être considérablement modifiés : renforcement par des enrochements supplémentaires et réhaussement de la cote d'arase (parfois de $2 \mathrm{~m}$ ) des brise-lames, mise en place d'un canal de recueil des eaux derrière la contre-digue. Deux solutions ont été dégagées pour assurer la stabilité de la face externe du brise-lames principal :

- l'une impliquant la pose de nouveaux accropodes de $12 \mathrm{~m}^{3}$ sur un tronçon de $100 \mathrm{~m}$; - l'autre, moins classique, permettant de conserver les accropodes de $9 \mathrm{~m}^{3}$ existants, à condition d'araser un haut-fond situé au pied de l'ouvrage, de façon à limiter le gonflement des vagues déferlant au pied de la digue principale sur cette zone critique.

Cette dernière option a été préférée. 


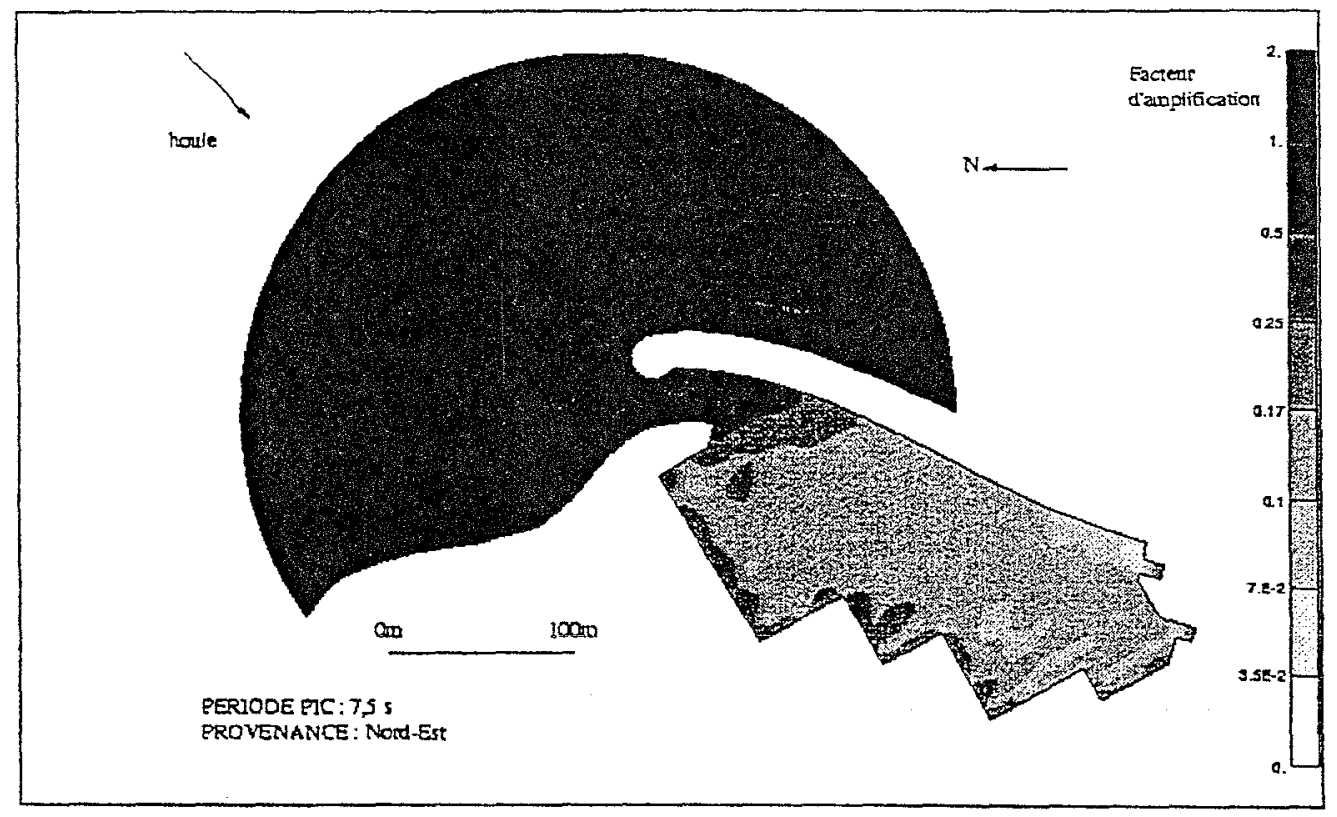

Figure 6: Facieur d'amplification par rapport à la hauteur au large. Configuration actuelle. Houle irrégulière. Tp $=7,5 \mathrm{~s}$. Provenance : $N E$

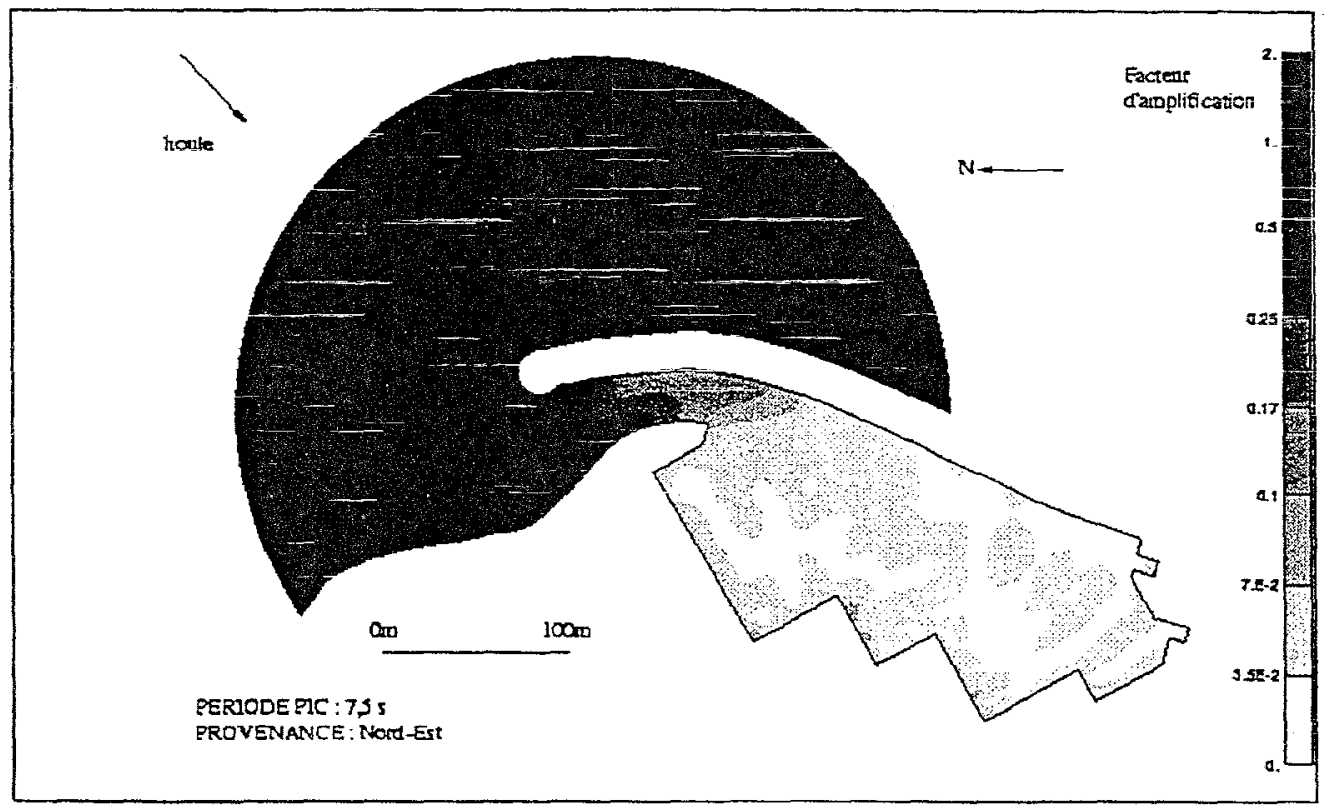

Figure 7 : Facteur d'amplification par rapport à la hauteur au large. Configuration future. Houle irrégulière. $T p=7,5$ s. Provenance : NE 


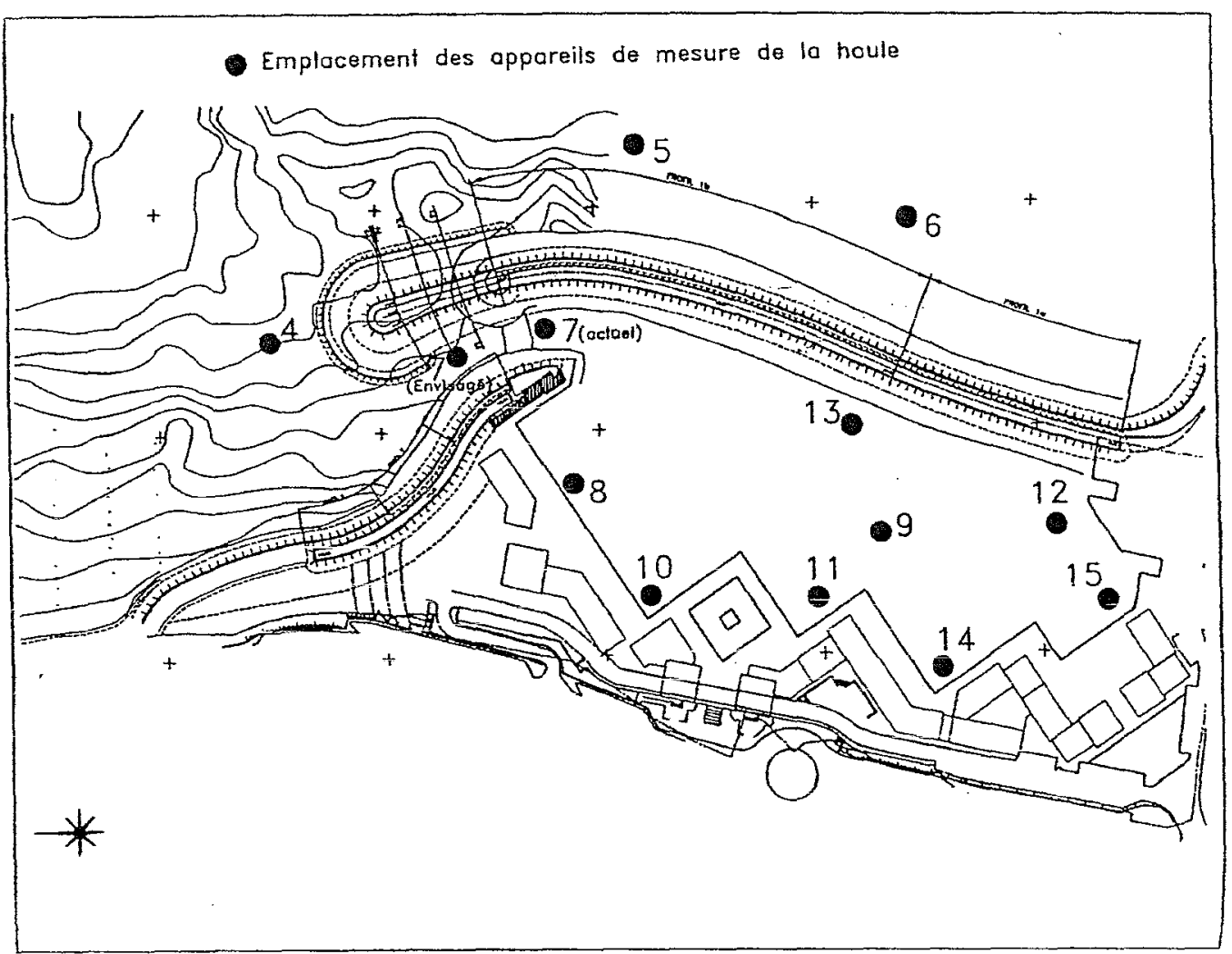

Figure 8 : Emplacements des points de mesure de l'agitation dans le modèle réduit physique

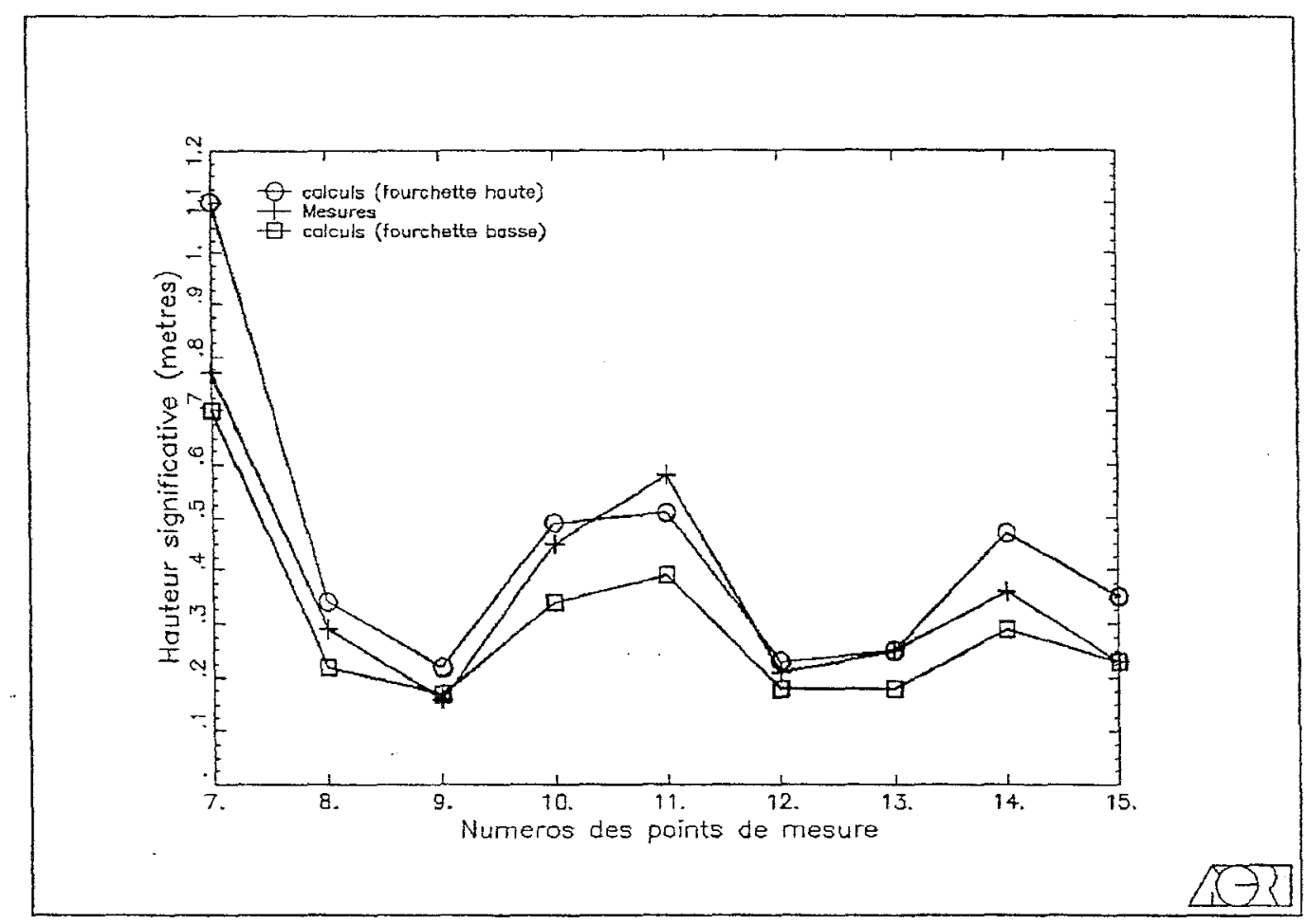

Figure 9: Comparaison des hauteurs de houle significatives calculées et mesurées. Configuration future. Houle irrégulière. $H_{s}$ au large $=5,7 \mathrm{~m} . \mathrm{Tp}=7,5 \mathrm{~s}$. Provenance : $N E$ 


\section{Conclusion}

Les simulations numériques en houle monochromatique ont permis d'estimer le niveau général d'agitation dans le port de Toga, laissant toutefois apparaitre de fortes résonances très pointues sur la bande des fréquences. On montre que les valeurs de ces agitations, localisées près des quais réfléchissants, sont très pessimistes par rapport à celles rencontrées en nature, étant donnée la dispersion en fréquences de la houle réelle.

Les hauteurs significatives calculées au moyen de simulations en houle irrégulière en admettant les processus gaussiens ont conduit à des valeurs très proches de celles mesurées en cuve à houle. Dans la perspective de nouvelles recherches, il conviendrait néanmoins d'affiner l'approche statistique pour estimer les hauteurs significatives dans le port (méthode upcrossing, degré de validité de la loi de répartition de Rayleigh).

Les études numériques ont permis de dégager une solution pour améliorer considérablement le confort et la sécurité du port de Toga. Mais, les tests en modèle réduit physique restent toutefois indispensables pour valider la solution et surtout s'assurer de la bonne tenue des ouvrages face à des phénomènes tels que les franchissements et le déferlement.

\section{Remerciements}

Les auteurs remercient vivement la Société du Port de Toga Plaisance, en particulier Mr Pont et Mr Louis, pour leur avoir confié la prise en charge de l'ensemble des études, ainsi que le laboratoire HR Wallingford pour leur excellente collaboration.

\section{Références}

[1] Berkhoff J.C.W. "Mathematical models for simple harmonic linear water waves. Wave diffraction and refraction", Publication $n^{\circ} 163$, Delft Hydraulic Laboratory, The Netherlands, 1976.

[2] Goda Y. "Random Waves and Spectra", Chapter 4 of Handbook of Coastal and Ocean Engineering, Volume 1 Wave Phenomena and Coastal Structures, 1990.

[3] Lajoie D. "Modelisation de la houle en zone côtière : Prévision de l'agitation à l'intérieur des ports et mise au point d'atténuateurs de houle dynamiques". Thèse de l'Université d'AixMarseille II, février 1996. 